\title{
Stellar dynamos with $\Omega \times J$ effect
}

\author{
V. V. Pipin ${ }^{1}$ and N. Seehafer ${ }^{2}$ \\ 1 Institute for Solar-Terrestrial Physics, Siberian Division of the Russian Academy of Sciences, 664033 Irkutsk, Russia \\ e-mail: pip@iszf.irk.ru \\ 2 Institut für Physik und Astronomie, Universität Potsdam, Karl-Liebknecht-Str. 24/25, 14476 Potsdam, Germany \\ e-mail: seehafer@uni-potsdam.de
}

Received 7 August 2008 / Accepted 4 November 2008

\begin{abstract}
Context. The standard dynamo model for the solar and stellar magnetic fields is based on the $\alpha \Omega$ mechanism, namely, an interplay between differential rotation (the $\Omega$ effect) and a mean electromotive force generated by helical turbulent convection flows (the $\alpha$ effect). There are, however, a number of problems with the $\alpha$ effect and $\alpha \Omega$ dynamo models. Two of them are that, in the case of the Sun, the obtained cycle periods are too short and the magnetic activity is not sufficiently concentrated at low latitudes.

Aims. We explore the role of turbulent induction effects that may appear in addition to the $\alpha$ effect. The additional effects result from the combined action of rotation and an inhomogeneity of the large-scale magnetic field. The best known of them is the $\boldsymbol{\Omega} \times \boldsymbol{J}$ effect. We also include anisotropic diffusion and a new dynamo term that is of third order in the rotation vector $\mathbf{\Omega}$.

Methods. We studied axisymmetric mean-field dynamo models containing differential rotation, the $\alpha$ effect, and the additional turbulent induction effects. The model calculations were carried out using the rotation profile of the Sun as obtained from helioseismic measurements and radial profiles of other quantities according to a standard model of the solar interior. In addition, we consider a dynamo model for a full sphere that is based solely on the joint induction effects of rotation and an inhomogeneity of the large-scale magnetic field, without differential rotation and the $\alpha$ effect (a $\delta^{2}$ dynamo model). This kind of dynamo model may be relevant for fully convective stars.

Results. With respect to the solar dynamo, the inclusion of the additional turbulent induction effects increases the period of the dynamo and brings the large-scale toroidal field closer to the equator, thus improving the agreement of the models with the observations. For the $\delta^{2}$ dynamo working in a full sphere, we find dynamo modes that are steady if the effect of anisotropic diffusion is not included. The inclusion of anisotropic diffusion yields a magnetic field oscillating with a period close to the turbulent magnetic diffusion time.
\end{abstract}

Key words. stars: magnetic fields - Sun: magnetic fields - magnetohydrodynamics (MHD)

\section{Introduction}

Most solar and stellar dynamo models use the scenario proposed by Parker $(1955,1979)$ where the magnetic field is produced by an interplay between differential rotation (the $\Omega$ effect) and the collective action of turbulent cyclonic convection flows, widely known as the $\alpha$ effect (Steenbeck et al. 1966; Krause \& Rädler 1980). This scheme suggests that the $\alpha$ effect is responsible for generating the poloidal component of the large-scale magnetic field (LSMF) of stars and other cosmic bodies.

There is an ongoing debate about a number of problems connected with the $\alpha$ effect and $\alpha \Omega$ dynamos (see, e.g., Ossendrijver 2003; Rüdiger \& Hollerbach 2004; Brandenburg \& Subramanian 2005). For instance, the period of the solar activity cycle poses a problem. Namely, for mixing-length estimates of the turbulent magnetic diffusivity in the convection zone and dynamo action distributed over the whole convection zone, the obtained cycle periods are generally much shorter than the observed $22 \mathrm{yr}$ period of the activity cycle. For thin-layer dynamos, the situation becomes even worse.

Furthermore, according to standard theory, the strength of the $\alpha$ effect follows roughly a $\cos \theta$ colatitude profile; that is, the effect is strongest near the poles. Similarly, also the variation in the solar rotation rate with radius, responsible for the $\Omega$ effect, is strongest at high latitudes (Schou et al. 1998). But solar magnetic activity in the form of active regions is mainly observed in latitudinal belts relatively close to the equator. As a possibility of bypassing this discrepancy, meridional (poloidal) flows are under discussion, leading to so-called flux-transport dynamos (see, e.g., Ossendrijver 2003; Rüdiger \& Hollerbach 2004; Dikpati \& Gilman 2007). Such flows may transport toroidal magnetic flux toward the equator and their speed may determine the cycle period.

In this paper, we consider the possible role of turbulent dynamo mechnisms that may complement the $\alpha$ effect or may be an alternative to it. Namely, according to mean-field dynamo theory, there are other turbulent sources of the LSMF besides the $\alpha$ effect in rotating electrically conducting fluids. The influence of the turbulence on the LSMF is expressed by the mean turbulent electromotive force (MEMF) $\mathcal{E}=\langle\boldsymbol{u} \times \boldsymbol{b}\rangle$, where $\boldsymbol{u}$ and $\boldsymbol{b}$ are the fluctuating parts of the velocity and magnetic field (angular brackets denote averages). Here we investigate the role of the $\boldsymbol{\Omega} \times \boldsymbol{J}$ effect (Rädler 1969) in axisymmetric mean-field dynamo models ( $\boldsymbol{\Omega}$ is the angular velocity of the stellar rotation and $\boldsymbol{J}$ the large-scale (or mean) electric-current density). This effect, which may be interpreted as resulting from an anisotropic turbulent electrical conductivity, has been investigated little in the context of solar and stellar dynamos. In the commonly used representation of the MEMF on the basis of symmetry arguments (see Rädler 1980; Krause \& Rädler 1980; Rädler 2000; Rädler et al. 2003), the $\boldsymbol{\Omega} \times \boldsymbol{J}$ effect represents a contribution to the $\delta$ term; another contribution to this term is the shear-current effect (Rogachevskii \& Kleeorin 2003, 2004). 


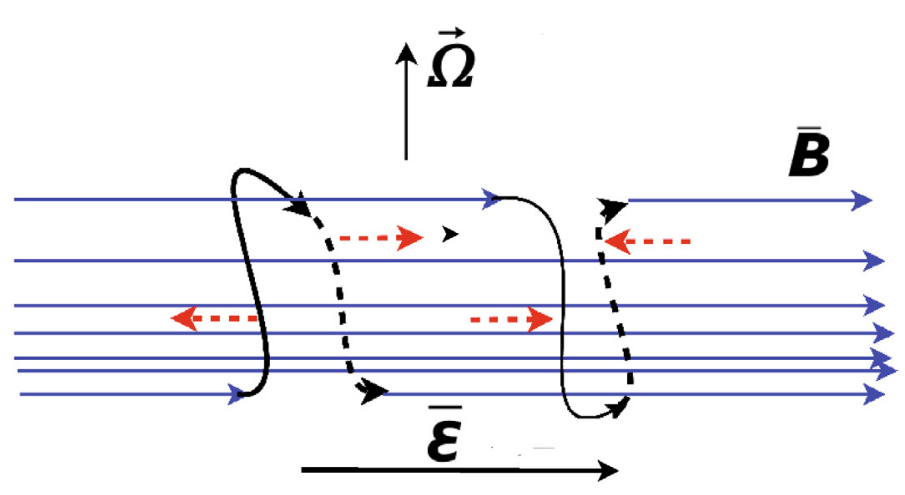

Fig. 1. Schematic of the $\boldsymbol{\Omega} \times \boldsymbol{J}$ effect.

An illustration of the $\boldsymbol{\Omega} \times \boldsymbol{J}$ effect is given in Fig. 1: the vector of the global rotation points in the vertical direction, and the horizontal field lines of a purely toroidal LSMF (solid blue lines) are linked by loop-like field lines (solid and dashed black lines) of a magnetic perturbation. The strength of the toroidal LSMF (represented by the field line density) decreases in the direction of the rotation vector. The $\boldsymbol{\Omega} \times \boldsymbol{J}$ effect comes about via the following steps. First, there is a contribution $(\boldsymbol{b} \cdot \nabla)\langle\boldsymbol{B}\rangle$ to the small-scale Lorentz force, giving rise to a velocity perturbation $\boldsymbol{u} \sim(\boldsymbol{b} \cdot \boldsymbol{\nabla})\langle\boldsymbol{B}\rangle$ parallel to the toroidal LSMF, indicated by dashed red arrows. Second, the Coriolis force deflects the velocity perturbation in the direction perpendicular to $\boldsymbol{\Omega}$ and $\langle\boldsymbol{B}\rangle$, that is, in the direction perpendicular the plane of the figure in the schematic (in the radial and latitudinal directions on the Sun or star). Third, the resulting deflected small-scale flow and the small-scale magnetic field give rise to a mean electromotive force $\mathcal{E} \sim\langle[(\boldsymbol{b} \cdot \boldsymbol{\nabla})\langle\boldsymbol{B}\rangle \times \boldsymbol{\Omega}] \times \boldsymbol{b}\rangle \sim-\left\langle\boldsymbol{b}^{2}\right\rangle(\boldsymbol{\Omega} \cdot \boldsymbol{\nabla})\langle\boldsymbol{B}\rangle$, where in the last step we have assumed that $\boldsymbol{b}$ is mainly parallel to $\boldsymbol{\Omega}$, so that a component of $\mathcal{E}$ parallel to $\boldsymbol{\Omega}$ could be neglected. The induced electromotive force is then parallel to the LSMF.

The MEMF was calculated analytically within a simplified version of the $\tau$ approximation (cf. Brandenburg \& Subramanian 2005) by Pipin (2008). The relevant part of the MEMF, containing the combined effects of rotation and a nonuniformity of the LSMF, reads

$$
\begin{aligned}
\mathcal{E}_{i}^{(d)}= & \left\{f_{1}^{(d)} e_{n} \bar{B}_{n i}+f_{2}^{(d)} \varepsilon_{i n m} \bar{B}_{m n}+\varepsilon f_{3}^{(d)} e_{i} e_{n} e_{m} \bar{B}_{m n}\right. \\
& +f_{1}^{(a)} \varepsilon_{i n m} e_{n} e_{l}\left(2 \varepsilon \bar{B}_{l m}-(\varepsilon+1) \bar{B}_{m l}\right) \\
& \left.+\varepsilon f_{4}^{(d)} e_{n} \bar{B}_{i n}\right\}\left\langle\boldsymbol{u}^{(0)^{2}}\right\rangle \tau_{\mathrm{c}},
\end{aligned}
$$

where $\boldsymbol{e}=\boldsymbol{\Omega} /|\boldsymbol{\Omega}|$ is the unit vector in the direction of the rotation vector, $\bar{B}_{i j}=\partial\left\langle B_{i}\right\rangle / \partial x_{j}$ the gradient tensor of the mean magnetic field (we here use Cartesian coordinates $x_{1}, x_{2}, x_{3}$ and the summation convention), $\boldsymbol{u}^{(0)}$ the small-scale or turbulent convective background velocity as present in the absence of rotation and a mean magnetic field, $\tau_{\mathrm{c}}$ the correlation time of $\boldsymbol{u}^{(0)}$, $\varepsilon=\sqrt{\left\langle\boldsymbol{b}^{(0)^{2}}\right\rangle} /\left(u_{\mathrm{c}} \sqrt{\mu_{0} \rho}\right)$ the square root of the ratio between the energies of a fluctuating magnetic background field $\boldsymbol{b}^{(0)}$, assumed to be generated by a small-scale dynamo, and the background velocity field $\boldsymbol{u}^{(0)}\left(u_{\mathrm{c}}=\sqrt{\left\langle\boldsymbol{u}^{(0)^{2}}\right\rangle}\right.$ is the rms value of the latter one and $\rho$ the mass density), and $f_{i}^{(d)}$ and $f_{i}^{(a)}(i=1,2, \ldots)$ denote functions of $\varepsilon$ and the Coriolis number $\Omega^{*}$ that are given in the Appendix. $\Omega^{*}$, measuring the influence of rotation on the turbulence, is defined by $\Omega^{*}=2 \Omega_{0} \tau_{\mathrm{c}}$, with $\Omega_{0}$ denoting the solid-body rotation rate; in our numerical calculations for the Sun we have chosen the value of the equatorial angular velocity at the solar surface for $\Omega_{0}$. Differential rotation, which gives rise to the shear-current effect, is not included at this place. We assume energy equipartition between the two background fields, i.e., $\varepsilon=1$.

By its nature, the assumed small-scale dynamo is fully independent of the mean field. An underlying assumption here is that developed turbulence in an electrically conducting, non-rotating fluid will be magnetohydrodynamic, rather than purely kinetic, if the small-scale motions are sufficiently complex. With rotation added, a mean-field dynamo can be superposed to the smallscale dynamo.

Hereafter, for simplicity, by the $\boldsymbol{\Omega} \times \boldsymbol{J}$ effect we mean all induction effects in Eq. (1) which are of odd order in $\boldsymbol{e}$, that is, the terms with coefficients $f_{1}^{(d)}, f_{3}^{(d)}$ and $f_{4}^{(d)}$, respectively. Of the remaining two terms, with coefficients $f_{2}^{(d)}$ and $f_{1}^{(a)}$, respectively, that with coefficient $f_{2}^{(d)}$ corresponds to the $\beta$ term in commonly used representations of $\mathcal{E}$ and describes an isotropic turbulent diffusion. The action of the term with coefficient $f_{1}^{(a)}$ will be referred to as anisotropic diffusion. The second part of this term, proportional to $f_{1}^{(a)}(\varepsilon+1)$, is known to describe an extra diffusion along $\boldsymbol{e}$ (Kitchatinov et al. 1994; Kitchatinov 2002, 2004).

The $\boldsymbol{\Omega} \times \boldsymbol{J}$ effect as it is usually understood is contained in the two source terms of first order in $\boldsymbol{e}$, with coefficients $f_{1}^{(d)}$ and $f_{4}^{(d)}$, respectively. Namely, in vector form the sum of these two terms can also be written as

$$
\begin{aligned}
& f_{1}^{(d)} \nabla(\boldsymbol{e} \cdot\langle\boldsymbol{B}\rangle)+\varepsilon f_{4}^{(d)}(\boldsymbol{e} \cdot \nabla)\langle\boldsymbol{B}\rangle= \\
& f_{1}^{(d)} \nabla(\boldsymbol{e} \cdot\langle\boldsymbol{B}\rangle)+\varepsilon f_{4}^{(d)}[\nabla(\boldsymbol{e} \cdot\langle\boldsymbol{B}\rangle)-\boldsymbol{e} \times(\nabla \times\langle\boldsymbol{B}\rangle)] \\
& =-\varepsilon f_{4}^{(d)} \boldsymbol{e} \times \mu_{0} \boldsymbol{J}+\left(f_{1}^{(d)}+\varepsilon f_{4}^{(d)}\right) \nabla(\boldsymbol{e} \cdot\langle\boldsymbol{B}\rangle)
\end{aligned}
$$

By their form, the two terms on the right-hand side of the last of Eqs. (2) correspond to the $\delta_{1}$ (here, $\boldsymbol{\Omega} \times \boldsymbol{J}$ ) and $\delta_{2}$ effects, respectively (cf. Krause \& Rädler 1980). In the axisymmetric case considered here, where gradients in the azimuthal direction vanish, the $\delta_{2}$ effect does not contribute to the important azimuthal component of the MEMF. Furthermore, it becomes a gradient, and its mean-field induction effect thus vanishes completely, if the Coriolis number $\Omega^{*}$ (i.e., the coefficient of $\nabla(\boldsymbol{e} \cdot\langle\boldsymbol{B}\rangle)$ ) does not vary spatially (as in a model considered in Sect. 3.2 below).

The term of third order in $\boldsymbol{e}$ in Eq. (1), with coefficient $f_{3}^{(d)}$, is an additional source term that does not seem to have been included in dynamo studies before. It involves only the symmetric part of the gradient tensor of the mean magnetic field (since $\left.e_{n} e_{m} \bar{B}_{m n}=e_{n} e_{m}\left(\bar{B}_{m n}+\bar{B}_{n m}\right) / 2\right)$ and the coefficient of this symmetric part, $\varepsilon f_{3}^{(d)} e_{i} e_{n} e_{m}$, is symmetric in the indices $m, n$. By its formal structure it thus belongs to the $\kappa$ term in respresentations of the MEMF as used in more recent papers of Rädler and his collaborators (see, e.g., Rädler 2000; Rädler et al. 2003; Rädler \& Stepanov 2006).

First results on the $\boldsymbol{\Omega} \times \boldsymbol{J}$ effect in mean-field dynamo models were, for instance, given in Rädler (1969), Stix (1976) and Krause \& Rädler (1980). At that time the dependence of the solar rotation rate on radius and latitude was not known well enough. In the early papers also the possibility of combining the $\alpha \Omega$ and $\delta \Omega$ mechanisms was discussed. Recently, the idea of a combination of the $\alpha$ and $\boldsymbol{\Omega} \times \boldsymbol{J}$ effects in dynamo models was anew 
suggested by Kitchatinov (2004). Here we explore axisymmetric kinematic $\alpha^{2} \delta \Omega$ dynamo models ( $\alpha$ effect plus $\boldsymbol{\Omega} \times \boldsymbol{J}$ effect plus differential rotation) for a convective spherical shell and for a full sphere, following the suggestion of Kitchatinov (2004) and using the calculations of Pipin (2008).

In the context of the model the following points concerning the $\boldsymbol{\Omega} \times \boldsymbol{J}$ effect are important: 1) the effect generates a MEMF along the LSMF, similar to the $\alpha$ effect; 2) the strength of the $\boldsymbol{\Omega} \times \boldsymbol{J}$ effect depends both on the intensity of the fluctuating magnetic fields and on their linkage with the LSMF. The latter contributes to the amount of magnetic and current helicities in the volume considered. Thus, the strength of the $\boldsymbol{\Omega} \times \boldsymbol{J}$ effect, as well as that of the $\alpha$ effect, is connected with the evolution of these quantities. In a companion study (Pipin 2007), the nonlinear saturation of the $\alpha$ and $\boldsymbol{\Omega} \times \boldsymbol{J}$ effects was investigated by integrating the mean-field equations coupled to an evolution equation for the small-scale current helicity forward in time. Similar to the nonlinear back-reaction of the mean magnetic field on the $\alpha$ effect, known as $\alpha$ quenching, the $\boldsymbol{\Omega} \times \boldsymbol{J}$ effect may be suppressed by strong mean fields. Here we concentrate on the linear-stability problem. Rather than numerically simulating time evolutions, the eigenvalues of the Jacobian matrix of the equations for the mean magnetic field are calculated directly. For a comparison of model calculations for the $\alpha$ effect and helicities in the solar convection zone, carried out using similar approximations as for the calculation of $\mathcal{E}$ in the repesent study, with observations at atmospheric levels, we refer to Kuzanyan et al. (2006).

We construct an $\alpha^{2} \delta \Omega$ dynamo model for the Sun with distributed dynamo action in the bulk of the convection zone. Such distributed dynamos have to be distinguished from boundarylayer dynamos that operate in the overshoot layer at the bottom of the convection zone, a thin transition region between the convection zone and the convectively stable radiative core, which is believed to coincide with the tachocline, where the differential rotation changes into rigid rotation in the radiative core. The main argument in favor of boundary-layer dynamos is that in the convection zone proper, due to the action of magnetic bouyancy, magnetic flux might not be stored long enough to allow the generation of a sufficiently strong toroidal field by differential rotation. This argument is based on the picture that the magnetic flux is concentrated in thin flux tubes (see, e.g., Spiegel \& Weiss 1980; Schüssler 1980; Galloway \& Weiss 1981; Schüssler \& Ferriz-Mas 2003). Large-scale magnetic fields are only little affected by magnetic bouyancy (Kichatinov \& Pipin 1993). A critical discussion of arguments for and against deep-seated and distributed dynamos, respectively, is found in Brandenburg (2005).

In addition, we develop a kinematic model of an axisymmetric $\delta^{2}$ dynamo in a full sphere. This dynamo is based solely on the turbulent electromotive force $\mathcal{E}^{(d)}$ given by Eq. (1). It might be working in fully convective stars. These objects presumably do not possess layers with a strong velocity shear like the tachoclines at the bottom of the convection zones of solartype stars, where the toroidal part of the large-scale magnetic field is believed to be generated. Nevertheless, fully convective stars are scarcely less magnetically active then stars with a radiative core and should, thus, harbor dynamo action. Discussions of the resulting dynamo problem may be found in the recent studies of Dobler et al. (2006), Chabrier \& Küker (2006) and Browning (2008). Mean-field dynamo models for fully convective stars have been mainly based on the $\alpha^{2}$ mechanism (Küker \& Rüdiger 1999; Elstner \& Rüdiger 2007; Chabrier \& Küker 2006). Also here, we suggest the $\boldsymbol{\Omega} \times \boldsymbol{J}$ effect (in its extended form) as a complement or an alternative to the $\alpha$ effect.
The remainder of the paper is organized as follows: in Sect. 2 we describe our models of the solar $\alpha^{2} \delta \Omega$ dynamo and the $\delta^{2}$ dynamo, as well as the used numerical procedure. Then, in Sect. 3 , we present the obtained results. Finally, in Sect. 4, we draw conclusions and discuss our results.

\section{Models and numerical procedure}

\subsection{The models}

We restrict ourselves to axisymmetric models. Mean fields may well be defined by azimuthal averages. Also, the large-scale solar magnetic field is largely axisymmetric; the deviations from axisymmetry, as they appear, for instance, in the form of active longitudes, are in general small. Nevertheless, a more comprehensive study would have to check whether our model is stable against non-axisymmetric perturbations.

The axisymmetric LSMF is represented in the form

$\langle\boldsymbol{B}\rangle=\operatorname{curl}\left(\frac{A \boldsymbol{e}_{\phi}}{r \sin \theta}\right)+B \boldsymbol{e}_{\phi}$

as the sum of a poloidal and a toroidal component; $A(r, \theta, t)$ and $B(r, \theta, t)$ are scalar functions of radius $r$, colatitude $\theta$ and time $t$, and $\boldsymbol{e}_{\phi}$ is the unit vector in the direction of the azimuthal coordinate $\phi$. The mean-field induction equation, containing the effects of differential rotation, expressed by the rotation rate $\Omega(r, \theta)=|\boldsymbol{\Omega}(r, \theta)|$, and of the MEMF, $\mathcal{E}$, then takes the form

$$
\begin{aligned}
& \frac{\partial A}{\partial t}=r \sin \theta \mathcal{E}_{\phi}, \\
& \frac{\partial B}{\partial t}=\frac{1}{r} \frac{\partial(\Omega, A)}{\partial(r, \theta)}+\frac{1}{r}\left(\frac{\partial r \mathcal{E}_{\theta}}{\partial r}-\frac{\partial \mathcal{E}_{r}}{\partial \theta}\right) .
\end{aligned}
$$

\subsubsection{The solar $\alpha^{2} \delta \Omega$ dynamo}

In the solar $\alpha^{2} \delta \Omega$ dynamo model, to keep the numerical effort (i.e., the spectral resolution, see Sect. 2.2 below) at a minimum, only the azimuthal $\boldsymbol{\Omega} \times \boldsymbol{J}$ effect is taken into account. This may be justified by the fact that the toroidal part of the solar LSMF is much stronger than the poloidal one. However, the other parts of the MEMF ( $\alpha$ effect, isotropic and anisotropic turbulent diffusion, turbulent pumping) are included in all components. Using the results of Pipin (2008), the components of the MEMF in spherical coordinates become

$$
\begin{aligned}
\mathcal{E}_{r}= & \tilde{\eta}_{\mathrm{T}}\left\{-\frac{f_{2}^{(d)}+(1+\varepsilon) f_{1}^{(a)} \sin ^{2} \theta}{r \sin \theta} \frac{\partial \sin \theta B}{\partial \theta}\right. \\
& +\frac{(1+\varepsilon) f_{1}^{(a)} \sin 2 \theta}{2 r} \frac{\partial r B}{\partial r}-G \sin 2 \theta f_{1}^{(a)} B \\
& +C_{\alpha}\left[G\left(f_{5}^{(a)} \cos ^{2} \theta+f_{10}^{(a)}+2 f_{6}^{(a)}\right) \frac{\cos \theta}{r^{2} \sin \theta} \frac{\partial A}{\partial \theta}\right. \\
& +U\left(f_{4}^{(a)} \cos ^{2} \theta+f_{11}^{(a)}+2 f_{8}^{(a)}\right) \frac{\cos \theta}{r^{2} \sin \theta} \frac{\partial A}{\partial \theta} \\
& +\left(f_{5}^{(a)} \cos ^{2} \theta+f_{6}^{(a)}-f_{7}^{(a)}\right) \frac{G}{r} \frac{\partial A}{\partial r} \\
& \left.\left.+\left(f_{4}^{(a)} \cos ^{2} \theta+f_{8}^{(a)}-f_{9}^{(a)}\right) \frac{U}{r} \frac{\partial A}{\partial r}\right]\right\}
\end{aligned}
$$




$$
\begin{aligned}
\mathcal{E}_{\theta}= & \tilde{\eta}_{\mathrm{T}}\left\{\frac{f_{2}^{(d)}+(1+\varepsilon) f_{1}^{(a)} \cos ^{2} \theta}{r} \frac{\partial r B}{\partial r}\right. \\
& -\frac{(1+\varepsilon) f_{1}^{(a)} \cos \theta}{r} \frac{\partial \sin \theta B}{\partial \theta} \\
& -\left[G f_{3}^{(a)}+(\varepsilon-1) U f_{2}^{(a)}+G\left(\cos ^{2} \theta-\sin ^{2} \theta\right) f_{1}^{(a)}\right] B \\
& -C_{\alpha}\left[\left(f_{5}^{(a)} \sin ^{2} \theta+f_{10}^{(a)}\right) \frac{G \cos \theta}{\sin \theta} \frac{\partial A}{\partial r}\right. \\
& +\left(f_{5}^{(a)} \cos ^{2} \theta+f_{6}^{(a)}+f_{7}^{(a)}\right) \frac{G}{r} \frac{\partial A}{\partial \theta} \\
& +\left(f_{4}^{(a)} \sin ^{2} \theta+f_{11}^{(a)}\right) \frac{U \cos \theta}{\sin \theta} \frac{\partial A}{\partial r} \\
& \left.\left.+\left(f_{4}^{(a)} \cos ^{2} \theta+f_{8}^{(a)}+f_{9}^{(a)}\right) \frac{U}{r} \frac{\partial A}{\partial \theta}\right]\right\},
\end{aligned}
$$

$$
\begin{aligned}
\mathcal{E}_{\phi}= & \frac{\tilde{\eta}_{\mathrm{T}}}{r \sin \theta}\left\{\left[f_{2}^{(d)}+f_{1}^{(a)}((1+\varepsilon)\right.\right. \\
& \left.\left.+(\varepsilon-1) \sin ^{2} \theta\right)\right] \frac{\partial^{2} A}{\partial r^{2}}+\left[f_{2}^{(d)}+f_{1}^{(a)}(2 \varepsilon\right. \\
& \left.\left.+(1-\varepsilon) \sin ^{2} \theta\right)\right] \frac{\sin \theta}{r^{2}} \frac{\partial}{\partial \theta} \frac{1}{\sin \theta} \frac{\partial A}{\partial \theta} \\
& +\frac{(1-\varepsilon) f_{1}^{(a)}}{r}\left(\frac{3 \sin 2 \theta}{2 r} \frac{\partial A}{\partial \theta}-\sin 2 \theta \frac{\partial^{2} A}{\partial r \partial \theta}\right. \\
& \left.+\sin ^{2} \theta \frac{\partial A}{\partial r}\right)+\sin 2 \theta f_{1}^{(a)}[(\varepsilon-1) U+\varepsilon G] \frac{\partial A}{\partial \theta} \\
& -\left[f_{1}^{(a)}\left(G-(G \varepsilon+(\varepsilon-1) U) \sin ^{2} \theta\right)\right. \\
& \left.+G f_{3}^{(a)}+(\varepsilon-1) U f_{2}^{(a)}\right] \frac{\partial A}{\partial r} \\
& +C_{\alpha} B r \sin 2 \theta G f_{12}^{(a)} \\
& \left.+C_{\delta} \varepsilon f_{4}^{(d)}\left(\frac{r \sin 2 \theta}{2} \frac{\partial B}{\partial r}-\sin ^{2} \theta \frac{\partial B}{\partial \theta}\right)\right\} .
\end{aligned}
$$

Here $G=(\partial / \partial r) \log \rho$ and $U=(\partial / \partial r) \log \left(u_{\mathrm{c}}^{2}\right)$ are the scale factors of density $(\rho)$ and turbulence intensity, respectively, and $\tilde{\eta}_{\mathrm{T}}=C_{\eta} \eta_{T}$, with $\eta_{T}=u_{\mathrm{c}} \ell_{\mathrm{c}} / 3$, where $\ell_{\mathrm{c}}$ denotes the correlation length of the background turbulence. $C_{\eta} \leq 1, C_{\alpha} \leq 1, C_{\delta} \leq 1$ are parameters to control the relative strengths of different turbulence effects. $C_{\alpha}$ and $C_{\delta}$ weight the $\alpha$ effect and the $\boldsymbol{\Omega} \times \boldsymbol{J}$ effect, respectively, and the Prandtl-like number $C_{\eta}$ regulates the turbulence level, with which all contributions are equally scaled. Below, we consider the case $C_{\eta}=1 / 5$.

A remark concerning the introduction of weighting factors for the $\alpha$ and $\boldsymbol{\Omega} \times \boldsymbol{J}$ effects seems to be in order. First, we wish to study the dynamo onset. For the conditions of the Sun, the two effects have therefore to be reduced in their strength. Second, the $\tau$ approximation, which is used to calculate $\mathcal{E}$, is based on heuristic closure assumptions for the turbulence; for a critical analysis of the approximation we refer to Rädler \& Rheinhardt (2007). Therefore, we have left a freedom to adjust at least the relative strengths of different turbulence effects.

The integration domain is radially bounded by $r=0.72 R_{\odot}$ and $r=0.96 R_{\odot}$, where the boundary conditions are $A=$ $0, \frac{\partial r B}{\partial r}=0$ at the bottom boundary (a usual approximation to perfect-conductor conditions, see, e.g., Köhler 1973; Jouve et al. 2008), and vacuum conditions (that is, $B=0$ and continuous match of the poloidal field component to an exterior potential field) at the top boundary.

In our numerical calculations we have used a dimensionless form of the equations, substituting $r=x R_{\odot}$ and $t \rightarrow \eta_{0} t / R_{\odot}^{2}$, where $\eta_{0}$ is the maximum value of $\eta_{T}$ in the convection zone. The estimated value for $\eta_{0}$ is $\sim 10^{9} \mathrm{~m}^{2} / \mathrm{s}$. The turbulent magnetic diffusion time on the basis of this value and the solar radius, our time unit, is about $15 \mathrm{yr}$. Thus, in order to match the solar conditions, the models should give cycle periods on the order of the turbulent diffusion time.

For the construction of the model the current knowledge of the rotation rate in the convection zone (Schou et al. 1998) was taken into account. In the numerical calculations, the rotation profile was approximated by (cf., e.g., Godier \& Rozelot 2000)

$\Omega(x, \theta)=\Omega_{0} f(x, \theta)$

with

$$
\begin{aligned}
f(x, \theta)= & \frac{1}{435}\left[435+50\left(x-x_{0}\right)+22 \phi(x)\left(1-5 \cos ^{2} \theta\right)\right. \\
& \left.-3.5\left(1-14 \cos ^{2} \theta+21 \cos ^{4} \theta\right)\right],
\end{aligned}
$$

where

$\phi(x)=0.5\left[1+\operatorname{erf}\left(50\left(x-x_{0}\right)\right)\right]$

and $x_{0}=0.71$ is the position of tachocline, situated below the bottom boundary of the integration domain.

The radial profiles of characteristic quantities of the turbulence, such as the rms value $u_{\mathrm{c}}$ and the correlation length and time $\ell_{\mathrm{c}}$ and $\tau_{\mathrm{c}}$ of the convective background velocity field, as well as the density stratification were calculated on the basis of a standard model of the solar interior (Stix 2002).

Some basic model quantities, namely, the differential rotation, the turbulent pumping velocity for the toroidal component of the LSMF and the radial profiles of the Coriolis number $\Omega^{*}$ and of $\alpha_{\phi \phi} \sec \theta$ ( $\alpha_{\phi \phi}$ measures the strength of the azimuthal $\alpha$ effect) are shown in Fig. 2 (see also Seehafer et al. 2003; Kuzanyan et al. 2006).

\subsubsection{The $\delta^{2}$ dynamo}

In our $\delta^{2}$ dynamo model for a full sphere, representing a star, differential rotation and the $\alpha$ effect are neglected. For simplicity, we assume a constant density stratification $R_{\star} G=-20$ throughout the star $\left(R_{\star}\right.$ is the radius of the star), corresponding to the value of $G$ at a distance of $R_{\star} / 2$ from the center of an M 5 dwarf with mass $M=0.2 M_{\odot}$, luminosity $L=0.0058 L_{\odot}$, radius $R_{\star}=0.23 R_{\odot}$ and surface temperature $T_{\text {surf }}=3458 \mathrm{~K}$ (we used the stellar evolution code TWIN (Eggleton 1971; Eggleton \& Kiseleva-Eggleton 2002) in the frontend-version WTTS (Izzard \& Glebbeek 2006)). We also neglect the effects of the spatial inhomogeneity of the turbulence and set $\tilde{\eta}_{\mathrm{T}}=\eta_{0}$, with $\eta_{0}$ denoting the value of the homogeneous turbulent magnetic diffusivity. Furthermore, we set $C_{\delta}=1$. At the surface vacuum boundary conditions are imposed.

Different from the treatment of the solar $\alpha^{2} \delta \Omega$ dynamo model in Sect. 2.1.1, now also the contributions of the $\boldsymbol{\Omega} \times \boldsymbol{J}$ effect to the $r$ and $\theta$ components of $\mathcal{E}$ are taken into account. The equations for the poloidal potential $A$ and for $\mathcal{E}_{\phi}$, Eqs. (4) and (8), remain unchanged (except for the omission of the term proportional to $C_{\alpha}$ and $U=0$ in Eq. (8)), while in the equation for the toroidal potential $B$, Eq. (5), the first term on the 

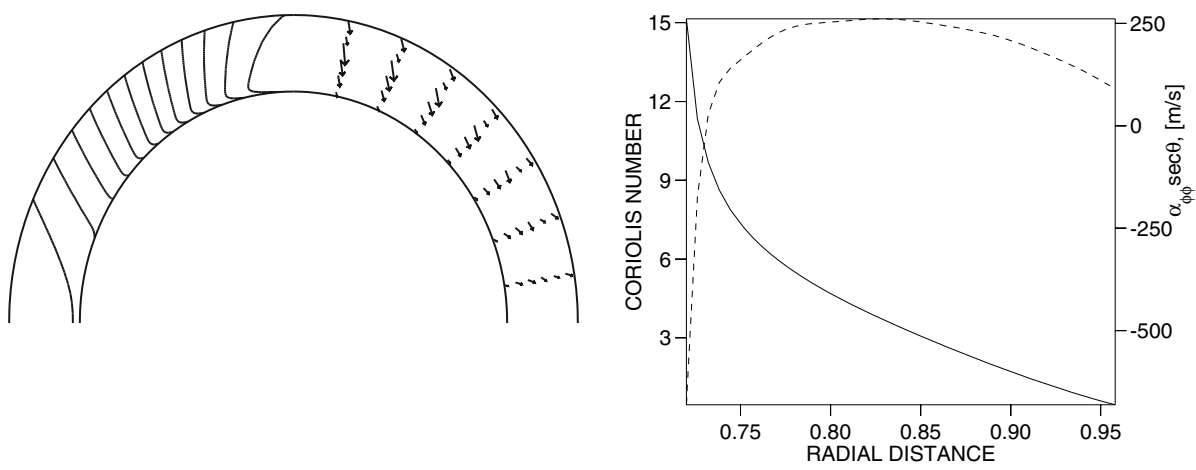

Fig. 2. Basic model quantities. Left panel: contours of the rotation rate in the solar convection zone (left) and geometry of the pumping velocity for the toroidal part of the LSMF (right). Right panel: radial profiles of the Coriolis number $\Omega^{*}$ (solid line) and $\alpha_{\phi \phi} \sec \theta$ (dashed line). $\alpha_{\phi \phi}$ changes sign near the bottom of the convection zone. right-hand side, describing the effect of the differential rotation, vanishes. We write the equation for $B$ in the form

$\frac{\partial B}{\partial t}=\frac{1}{r}\left(\frac{\partial r\left(\mathcal{E}_{\theta}+\mathcal{E}_{\theta}^{(\delta)}\right)}{\partial r}-\frac{\partial\left(\mathcal{E}_{r}+\mathcal{E}_{r}^{(\delta)}\right)}{\partial \theta}\right)$,

where $\mathcal{E}_{r}$ and $\mathcal{E}_{\theta}$ are given by Eqs. (6) and (7) in Sect. 2.1.1 (with $\left.\tilde{\eta}_{\mathrm{T}}=\eta_{0}, U=0, C_{\alpha}=0\right)$ and $\mathcal{E}_{r}^{(\delta)}$ and $\mathcal{E}_{\theta}^{(\delta)}$ denote the additional contributions due to the $\boldsymbol{\Omega} \times \boldsymbol{J}$ effect. For these we have

$$
\begin{aligned}
\mathcal{E}_{r}^{(\delta)}= & \eta_{0} \varepsilon\left\{\frac{\cos \theta\left(c_{3} f_{3}^{(d)} \cos 2 \theta+f_{4}^{(d)}\right)}{r^{2} \sin \theta}\left(\frac{\partial^{2} A}{\partial r \partial \theta}-\frac{1}{r} \frac{\partial A}{\partial \theta}\right)\right. \\
& +\frac{c_{3} f_{3}^{(d)} \cos ^{2} \theta}{r} \frac{\partial^{2} A}{\partial r^{2}} \\
& \left.-\frac{\left(c_{3} f_{3}^{(d)} \cos ^{2} \theta+f_{4}^{(d)}\right)}{r^{2}}\left(\frac{1}{r} \frac{\partial^{2} A}{\partial \theta^{2}}+\frac{\partial A}{\partial r}\right)\right\},
\end{aligned}
$$

$$
\begin{aligned}
\mathcal{E}_{\theta}^{(\delta)}= & \eta_{0} \varepsilon\left\{\frac{\left(f_{4}^{(d)}-c_{3} f_{3}^{(d)} \cos 2 \theta\right)}{r^{2}}\left(\frac{\partial^{2} A}{\partial r \partial \theta}-\frac{1}{r} \frac{\partial A}{\partial \theta}\right)\right\} \\
& +\frac{\sin 2 \theta}{2 r^{2}} c_{3} f_{3}^{(d)}\left(\frac{1}{r} \frac{\partial^{2} A}{\partial \theta^{2}}+\frac{\partial A}{\partial r}\right) \\
& \left.-\frac{\cos \theta\left(f_{4}^{(d)}+c_{3} f_{3}^{(d)} \sin ^{2} \theta\right)}{r \sin \theta} \frac{\partial^{2} A}{\partial r^{2}}\right\} .
\end{aligned}
$$

$c_{3}$ is a weighting factor for the component of the $\boldsymbol{\Omega} \times \boldsymbol{J}$ effect with coefficient $f_{3}^{(d)}$ (i.e., the component of third order in $\boldsymbol{e}$ ). There is no dynamo with $c_{3}=1$. The meaning of this coefficient for the model will be explained further in Sect. 3.2 below. Dynamo action sets in when both $c_{3}$ and the Coriolis number exceed threshold values. In our numerical calculations for this model, length and time are normalized to $R_{\star}$ and $R_{\star}^{2} / \eta_{0}$, respectively.

\subsection{The numerical procedure}

The eigenvalue problem is treated by means of a Galerkin method. We seek the solutions to Eqs. (4), (5) for the $\alpha^{2} \delta \Omega$ dynamo and to Eqs. (4), (12) for the $\delta^{2}$ dynamo in the form

$$
\begin{aligned}
& A(x, \theta, t)=\mathrm{e}^{\lambda t} \sum_{n} \sum_{m} a_{n m} \sin \theta S_{n m}^{(A)}(x) P_{m}^{1}(\cos \theta), \\
& B(x, \theta, t)=\mathrm{e}^{\lambda t} \sum_{n} \sum_{m} b_{n m} S_{n}^{(B)}(x) P_{m}^{1}(\cos \theta),
\end{aligned}
$$

where $S_{n m}^{(A)}$ and $S_{n}^{(B)}$ are linear combinations of Legendre polynomials and $P_{m}^{1}$ is the associated Legendre function of degree $m$ and order 1. By these expansions the regularity of the solutions at the poles $\theta=0$ and $\theta=\pi$, where both $A$ and $B$ are set to zero, is ensured. To satisfy the conditions at the radial boundaries, we use the "basis recombination" of the Legendre polynomials (see Boyd 2001). In the case of the solar $\alpha^{2} \delta \Omega$ dynamo this reads

$S_{n m}^{(A)}(x)=P_{n}(x)+a_{1} P_{n+1}(x)+a_{2} P_{n+2}(x)$,

$S_{n}^{(B)}(x)=P_{n}(x)+b_{1} P_{n+1}(x)+b_{2} P_{n+2}(x)$,

where $P_{n}(x)$ is the Legendre polynomial of degree $n$ and

$$
\begin{array}{ll}
a_{1}=\frac{2 n+3}{(n+2)^{2}+2 m}, & a_{2}=-\frac{(n+1)^{2}+2 m}{(n+2)^{2}+2 m}, \\
b_{1}=-\frac{4 n+4}{2 n^{2}+6 n+3}, & b_{2}=-\frac{2 n^{2}+2 n-1}{2 n^{2}+6 n+3} .
\end{array}
$$

For the $\delta^{2}$ dynamo we exploit the symmetry of the problem, as $(x, \theta)$ and $(-x, \theta+\pi)$ represent the same point. The boundary conditions on $x$ are then satisfied with

$$
\begin{aligned}
& S_{n m}^{(A)}(x)=x\left(P_{n}(x)-\frac{2+2 m+n(n+1)}{2+2 m+(n-2)(n-1)} P_{n-2}(x)\right), \\
& S_{n}^{(B)}(x)=x\left(P_{n}(x)-P_{n-2}(x)\right),
\end{aligned}
$$

and the summations in Eqs. (15), (16) run over even $m+n$ only and start from $n=2$.

Integrations over radius and latitude, necessary to calculate the expansion coefficients $a_{n m}$ and $b_{n m}$, were done by means of the Gauss-Legendre procedure. The used computer code was developed employing the free computer algebra system Maxima, and the eigenvalue problem was solved with the help of Lapack routines, which are accessible within Maxima. For the solar dynamo problem we used the first 7 modes in the radial expansion and the first 20 modes in the latitudinal expansion, while in the case of the $\delta^{2}$ dynamo a decomposition with $16 \times 16$ modes was applied. The results were confirmed by a number of runs with higher resolutions.

\section{Results}

\subsection{The solar $\alpha^{2} \delta \Omega$ dynamo}

\subsubsection{Effects of anisotropic diffusion and the small-scale dynamo in the $\alpha^{2} \Omega$ dynamo}

From Eq. (1) or Eqs. (6)-(8) it is seen that for the case of energy equipartition, $\varepsilon=1$, the small-scale dynamo makes significant contributions to different parts of the MEMF. Kitchatinov (2002, 2004) has found that anisotropic diffusion may be in a large part responsible for the equatorial drift of the toroidal LSMF. This drift is actually an extra diffusion (of both the toroidal and 

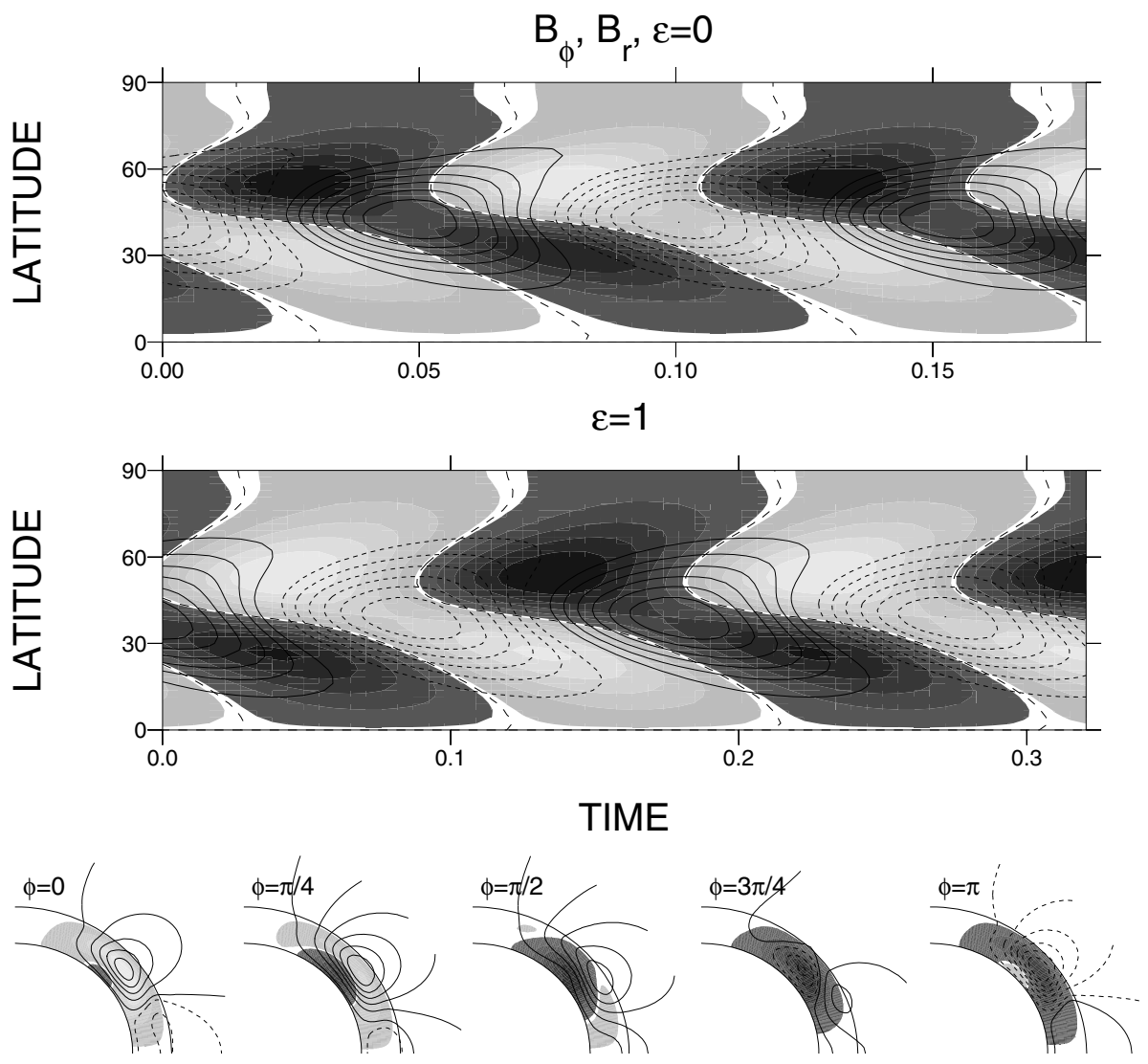

Fig. 3. Effects of anisotropic diffusion and the small-scale dynamo for an $\alpha^{2} \Omega$ dynamo model on the basis of the first unstable dipolar eigenmode $\left(C_{\alpha}=0.087\right)$. Top and middle: butterfly diagrams in the form of isocontours of the toroidal LSMF (integrated over depth in the convection zone) and overlaid greyscale plots of the radial LSMF at the top boundary without (top) and with (middle) the effects of anisotropic diffusion and the small-scale dynamo. Time is measured in units of $R_{\odot}^{2} / \eta_{0}$. Solid/dashed lines and bright/dark areas indicate positive/negative field values. Bottom: snapshots of the strength of the toroidal LSMF (greyscale plot) and field lines of the poloidal LSMF over half a cycle for the model containing the effects of anisotropic diffusion and the smallscale dynamo. Solid/dashed lines indicate clockwise/counter-clockwise field direction along the poloidal field lines. poloidal parts of the mean field) along the rotation axis $\boldsymbol{e}$. In Eq. (1) it is described by the second part of the anisotropicdiffusion term, which is proportional to $f_{1}^{(a)}(\varepsilon+1)$. Thus, for $\varepsilon=1$ the drift is enhanced by a factor of two compared to the case of $\varepsilon=0$. The first part of the anisotropic-diffusion term in Eq. (1) can in vector form be written as $2 f_{1}^{(a)} \varepsilon \boldsymbol{e} \times \nabla(\boldsymbol{e} \cdot\langle\boldsymbol{B}\rangle)$. Due to the assumed axisymmetry, it is purely toroidal (azimuthal). Therefore, it does not influence the toroidal part of the mean field. It actually describes a diffusion of the poloidal part of the mean field in the direction perpendicular to the rotation vector $\boldsymbol{e}$.

In Fig. 3 (top and middle), showing butterfly diagrams in the form of isocontours of the toroidal LSMF (integrated over depth in the convection zone) and overlaid greyscale plots of the radial LSMF at the top boundary, the effects of anisotropic diffusion and the small-scale dynamo are demonstrated for a pure $\alpha^{2} \Omega$ dynamo model on the basis of the first unstable dipolar eigenmode. By dipolar/quadrupolar modes we mean modes that are antisymmetric/symmetric with respect to the equatorial plane. The used dipolar mode is not the primary dynamo eigenmode, see Sect. 3.1.2 below. Qualitatively, the results presented here resemble those of Kitchatinov (2002), who did not include the effect of the small-scale dynamo and used a slightly different rotation law.

As is seen in Fig. 2 (left), the direction of the turbulent pumping velocity is such that it transports magnetic flux toward the equator. So also the pumping effect offers a possibility to explain the observed latitudinal distribution and drift of the solar activity phenomena (Guerrero \& de Gouveia Dal Pino 2008). In our model the pumping effect is obviously to weak to produce a noticeable equatorward drift of the toroidal LSMF.

Finally, as is seen in Fig. 3 (bottom), the dynamo wave moves mainly radially outward. This is in accordance with the tendency of the $\alpha \Omega$ dynamo waves to move along isorotation surfaces (Yoshimura 1975). In the bulk of the convection zone these surfaces are largely parallel to radius (cf. Fig. 2, left panel). A better agreement with the observations could thus be expected if the toroidal LSMF were confined to the tachocline, where the rotation rate varies mainly with radius. This would then amount to a boundary-layer dynamo, which is basically different from the distributed convection-zone dynamo considered here. A more complete model of the solar dynamo would include the dynamo effects of both the tachocline and the convection zone proper.

\subsubsection{The $\alpha^{2} \delta \Omega$ dynamo}

Figure 4 (left) shows a linear-stability diagram for the $\alpha^{2} \delta \Omega$ dynamo, i.e., the stability boundary for the most unstable eigenmode in the $C_{\delta}-C_{\alpha}$ plane. The $\alpha^{2} \Omega$ dynamo is included as a limiting case $\left(C_{\delta}=0\right)$. In the figure, regions where the eigenmode with the largest growth rate is a dipolar mode are indicated by shading. In the limiting case of $C_{\delta}=0$ the dipolar modes are not the most unstable modes. Though a dipolar mode becomes unstable only slightly above the total-stability boundary, there is a preference for quadrupolar modes in the pure $\alpha^{2} \Omega$ dynamo, contrary to the antisymmetric parity characterizing the large-scale solar magnetic field. The parity issue is met in other dynamo models, like, e.g., the flux-transport models, as well (see, e.g., Dikpati et al. 2005). For the pure $\delta \Omega$ dynamo $(\boldsymbol{\Omega} \times \boldsymbol{J}$ effect plus differential rotation), on the other hand, there is a preference of dipolar modes ( $C_{\alpha}=0$ in Fig. 4). These are, however, non-oscillatory. An example of such a mode is shown in Fig. 4 (right). The fact that in the pure $\delta \Omega$ model only non-oscillatory modes are found seems to indicate that for models of the solar dynamo the $\alpha$ effect is also needed, at least for models without meridional circulation. 


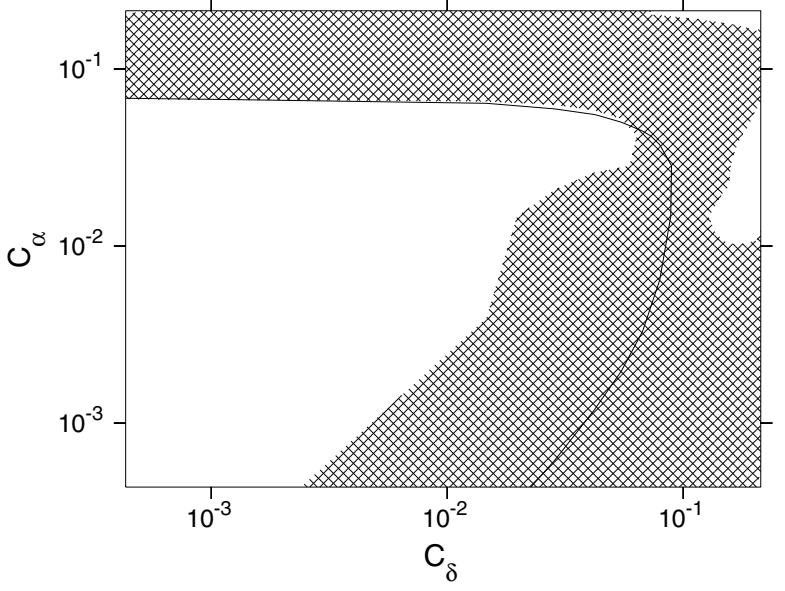

For increasing $C_{\alpha}$, oscillatory dipolar modes are excited if $C_{\alpha}$ is no longer very small compared to $C_{\delta}$. The properties of these modes are determined by the ratio $C_{\alpha} / C_{\delta}$. Figure 5 shows a simulated butterfly diagram (isocontours of the toroidal LSMF with an overlaid greyscale plot of the radial LSMF at the top boundary) for $C_{\delta}=0.07, C_{\alpha}=0.01$. For these parameter values we obtain a slow dynamo wave propagating from the equator to the pole for both the toroidal and poloidal fields, in contrast to the solar observations. The period of the dynamo is considerably increased compared to the pure $\alpha^{2} \Omega$ dynamo shown in Fig. 3 .

In the intermediate case, when the $\boldsymbol{\Omega} \times \boldsymbol{J}$ effect exceeds the $\alpha$ effect while the latter one is not very small compared to the first one, we find a good match with the solar case. A corresponding butterfly diagam and snapshots of the toroidal and poloidal fields over half a cycle for $C_{\delta}=0.1, C_{\alpha}=0.05$ are shown in Fig. 6. While the wings of the obtained dynamo waves are too wide, other qualitative properties, such as the directions of the drifts of the toroidal and poloidal parts of the magnetic field and their phase relation (e.g., polar reversal of $B_{r}$ shortly after maximum of $B_{\phi}$ at low latitudes) are in good agreement with the observations. The period of the dynamo is shorter than but comparable with the turbulent magnetic diffusion time. In the range $1<C_{\delta} / C_{\alpha}<3$ the match with the solar observations is best (it can be seen in Fig. 4 that in this range the dipolar modes are dominating at the stability boundary).

The obtained ratio $B_{\mathrm{T}} / B_{\mathrm{P}}$ of the toroidal $\left(B_{\mathrm{T}}\right)$ and poloidal $\left(B_{\mathrm{P}}\right)$ field strengths is about 50 , the ratio of the toroidal and poloidal field energies thus on the order of $10^{3}$. This comes close to the ratio $B_{\mathrm{T}} / B_{\mathrm{P}} \sim 100$ estimated from measurements of the fields in active regions (yielding the estimate $B_{\mathrm{T}} \sim 200 \mathrm{G}$ for a distributed toroidal field in the convection zone, cf. Stix 2002, Sect. 8.4.1) and the field strength at the solar poles (yielding the estimate $B_{\mathrm{P}} \sim 1 \mathrm{G}$ for the poloidal field).

\subsection{The $\delta^{2}$ dynamo}

The usual $\boldsymbol{\Omega} \times \boldsymbol{J}$ effect, given by the term $-\varepsilon f_{4}^{(d)} \boldsymbol{e} \times \mu_{0} \boldsymbol{J}$ in the expression for the turbulent emf (cf. Eq. (2)), does not contribute to $\mathcal{E} \cdot \boldsymbol{J}$. Therefore, it cannot bring energy into the mean magnetic field and is not capable of dynamo action when working alone. It can yield a dynamo, however, when acting together with differential rotation. The role of the effect is then to transfer energy from the toroidal to the poloidal field. In our model of the $\delta^{2} \mathrm{dy}-$ namo differential rotation is neglected (as well as the $\alpha$ effect). Energy has thus to be provided by the additional source term in

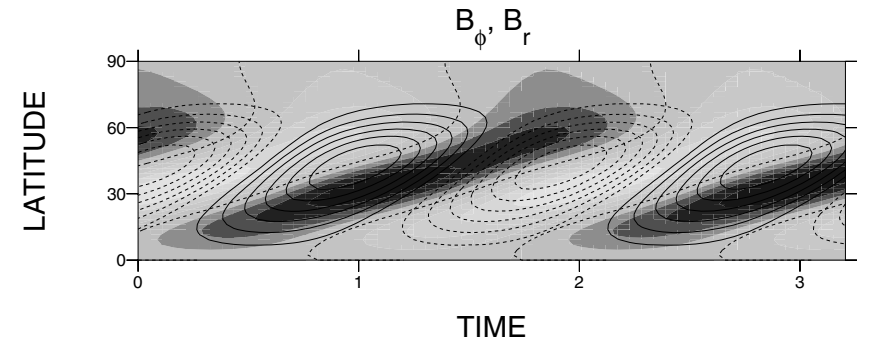

Fig. 5. Butterfly diagram in the form of isocontours of the toroidal LSMF (integrated over depth in the convection zone) and overlaid greyscale plot of the radial LSMF at the top boundary for an $\alpha^{2} \delta \Omega$ dynamo with $C_{\delta}=0.07, C_{\alpha}=0.01$. Time is measured in units of $R_{\odot}^{2} / \eta_{0}$. Solid/dashed lines and bright/dark areas indicate positive/negative field values.

our model, the term of third order in $\boldsymbol{e}$. Before studying our axisymmetric model of the $\delta^{2}$ dynamo in spherical geometry, we consider a stronger simplified model in plane geometry, in order to check whether such a dynamo is in principle possible. For this purpose, Eq. (1) is simplified to

$\mathcal{E}=-\eta_{0} \nabla \times\langle\boldsymbol{B}\rangle+c_{1}(\boldsymbol{e} \cdot \nabla)\langle\boldsymbol{B}\rangle+c_{3} \boldsymbol{e}(\boldsymbol{e} \cdot \nabla)(\boldsymbol{e} \cdot\langle\boldsymbol{B}\rangle)$,

where $c_{1}$ and $c_{3}$ are constants. This MEMF contains an isotropic diffusion term and two terms corresponding to the $\boldsymbol{\Omega} \times \boldsymbol{J}$ effect, one of first order and the other of third order in $\boldsymbol{e}$ (their formal structures agree with those of the terms with coefficients $f_{4}^{(d)}$ and $f_{3}^{(d)}$, respectively, in Eq. (1)). The rotation axis coincides with the $z$ axis of a system of Cartesian coordinates $x, y, z$, and the mean magnetic field is written as

$\langle\boldsymbol{B}\rangle=\nabla \times\left(a(x, z) \boldsymbol{e}_{y}\right)+b(x, z) \boldsymbol{e}_{y}$.

$\boldsymbol{e}_{y}$ is the unit vector in the $y$ direction, in which the system is invariant (corresponding to the $\phi$ direction in the spherical model). From the mean-field induction equation

$\frac{\partial\langle\boldsymbol{B}\rangle}{\partial t}=\nabla \times \mathcal{E}$

we then get

$\left\{\frac{\partial}{\partial t}-\eta_{0}\left(\frac{\partial}{\partial x^{2}}+\frac{\partial}{\partial z^{2}}\right)\right\} a=c_{1} \frac{\partial b}{\partial z}$,

$\left\{\frac{\partial}{\partial t}-\eta_{0}\left(\frac{\partial}{\partial x^{2}}+\frac{\partial}{\partial z^{2}}\right)\right\} b=-c_{1} \frac{\partial^{3} a}{\partial z^{3}}-\left(c_{1}+c_{3}\right) \frac{\partial^{3} a}{\partial x^{2} \partial z}$. 


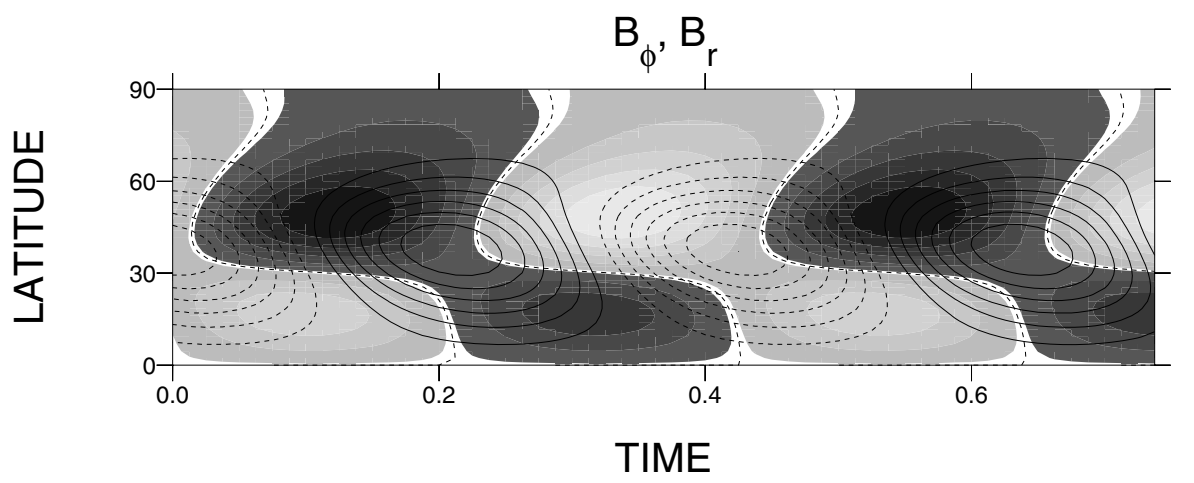

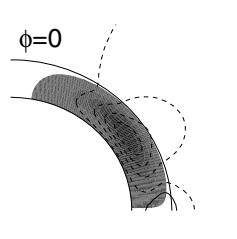
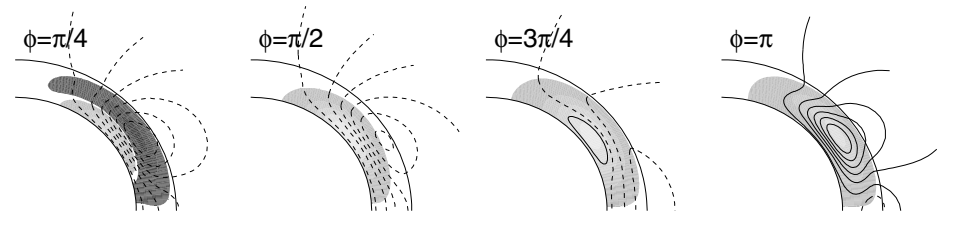

Solutions to Eqs. (26), (27) may be sought in the form

$a=\hat{a} \exp \{\gamma t+\mathrm{i}(k x+q z)\}, \quad b=\hat{b} \exp \{\gamma t+\mathrm{i}(k x+q z)\}$,

with $\gamma$ being determined by the condition that the determinant of the resulting system of algebraic equations vanishes, that is,

$\left[\gamma+\eta_{0}\left(k^{2}+q^{2}\right)\right]^{2}+q^{2}\left[c_{1}^{2}\left(k^{2}+q^{2}\right)+c_{1} c_{3} k^{2}\right]=0$,

with roots

$\gamma_{1,2}=-\eta_{0}\left(k^{2}+q^{2}\right) \mp \sqrt{-q^{2}\left[c_{1}^{2}\left(k^{2}+q^{2}\right)+c_{1} c_{3} k^{2}\right]}$.

It is seen that $\gamma$ is real for growing modes, so that only nonoscillatory dynamo modes can be excited. Furthermore, necessary, but not sufficient conditions for the existence of a dynamo mode are (i) $c_{1} \neq 0$; (ii) $c_{3} \neq 0$; (iii) $k \neq 0$; (iv) $q \neq 0$ and (v)

$$
-\frac{c_{3}}{c_{1}}>\frac{k^{2}+q^{2}}{k^{2}} \text {. }
$$

That is, $c_{1}$ and $c_{3}$ must have opposite signs and the absolute value of $c_{3}$ must be larger than that of $c_{1}$. Now in the limit of fast rotation, $\Omega^{*} \gg 1$, the signs of $f_{3}^{(d)}$ and $f_{4}^{(d)}$ are opposite, namely, for $\Omega^{*} \rightarrow \infty$ one gets $f_{3}^{(d)} / f_{4}^{(d)} \rightarrow-2$. Suppose $c_{1}=-c_{3} / 2$. Then the (necessary and sufficient) condition for dynamo instability becomes

$\frac{c_{3}^{2} q^{2}\left(k^{2}-q^{2}\right)}{4 \eta_{0}^{2}\left(k^{2}+q^{2}\right)^{2}}>1$.

It can be satisfied if $|k|>|q|$. The optimal wave number ratio is $|k| /|q|=\sqrt{3}$, giving dynamo excitation for $c_{3}^{2}>32 \eta_{0}^{2}$. These findings are largely confirmed by the kinematic $\delta^{2}$ dynamo model in spherical geometry, for which we now discuss the eigenmodes of dipolar type.

In the spherical model we have left only one of the above two parameters, $c_{3}$, as a weighting factor for the term with coefficient $f_{3}^{(d)}$ (term of third order in $\boldsymbol{e}$ ), see Eqs. (13), (14). Besides that we now also vary the Coriolis number $\Omega^{*}$. If we neglect the effects of anisotropic turbulent diffusion (but not those of isotropic turbulent diffusion) and density stratification (i.e., turbulent pumping) in the spherical model, we get steady dynamo modes as obtained in the plane model above. Stability diagrams
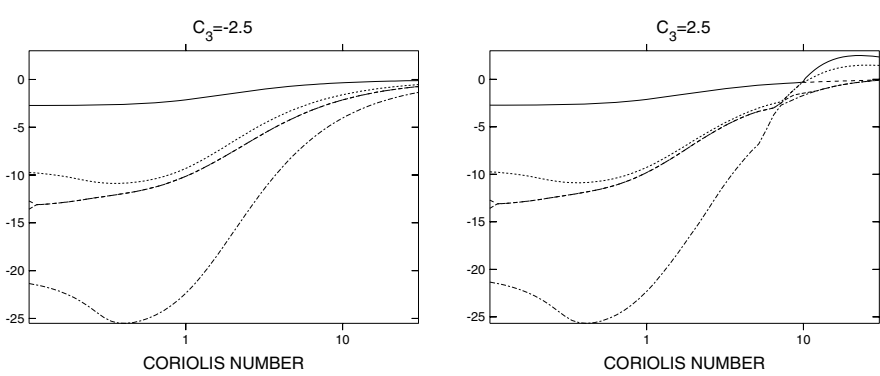

Fig. 7. Stability diagrams for the $\delta^{2}$ dynamo in spherical geometry without the effects of anisotropic diffusion and turbulent pumping for $c_{3}=-2.5$ (left) and $c_{3}=2.5($ right $)$. The four largest real parts of the eigenvalues $\lambda$ are shown as functions of the Coriolis number. Only dipolar modes are considered.

for this case are shown in Fig. 7, where the left panel shows that there is no dynamo effect if the coefficients of the terms describing the $\boldsymbol{\Omega} \times \boldsymbol{J}$ effect do not have signs in accordance with what is expected to be necessary for dynamo action from the consideration of the plane model. Namely, $f_{3}^{(d)}$ is negative and $f_{4}^{(d)}$ positive for all $\Omega^{*},\left|f_{3}^{(d)} / f_{4}^{(d)}\right|$ monotonically increasing with $\Omega^{*}$ and $f_{3}^{(d)} / f_{4}^{(d)} \approx-2$ for $\Omega^{*} \gtrsim 5$. For $c_{3}=1$ we found no dynamo instability in the whole $\Omega^{*}$ interval. The condition for dynamo action was found to be $c_{3}>2$. Furthermore, a threshold value of $\Omega^{*}$ must be exceeded. The larger $c_{3}$, the smaller the threshold value of $\Omega^{*}$.

Including anisotropic turbulent diffusion decreases the threshold values of $c_{3}$ and $\Omega^{*}$ for instability, while including turbulent transport (pumping), associated with density stratification, raises them. Yet, including one of them or both together changes the character of the dynamo bifurcation from a steady-state to a Hopf bifurcation. Namely, the two most unstable modes then merge, yielding a slowly oscillating dynamo mode. "Slowly" here means that the time period of the mode is larger than the turbulent diffusion time of the system. For the parameter choice $c_{3}=2.5, R_{\star} G=-20$, the threshold value of the Coriolis number is $\Omega^{*} \approx 9.1$, and the frequency of the obtained dynamo mode is $\omega \approx 0.8$ inverse diffusion times. Figure 8 shows the evolution of the large-scale toroidal and poloidal field components over half a cycle. It is seen that the strongest toroidal and poloidal fields are concentrated close to the rotation axis in the 


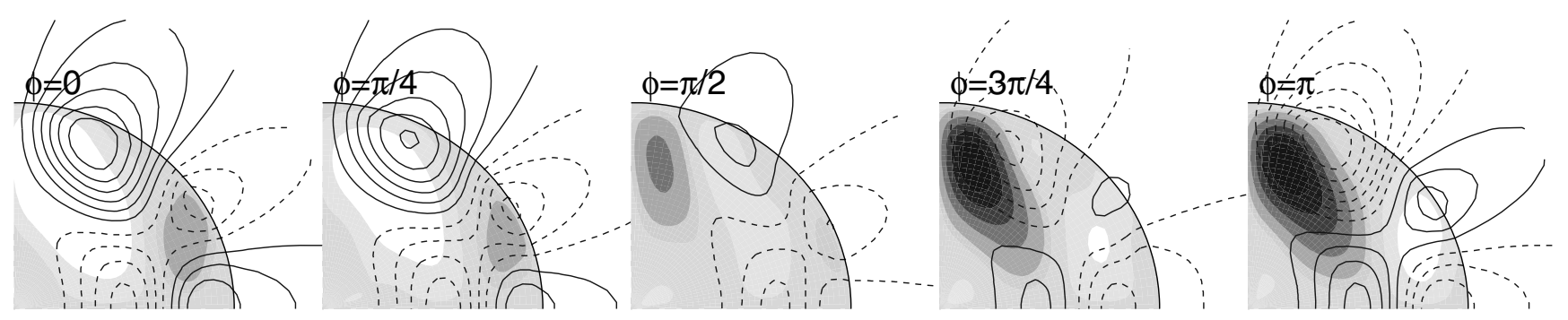

Fig. 8. Snapshots of the magnetic field in the time-periodic $\delta^{2}$ dynamo (first unstable mode) over half a cycle for $c_{3}=2.5, \Omega^{*}=9.1$ in the form of greyscale plots for the toroidal LSMF (bright/dark areas indicate positive/negative field values) and field lines of the poloidal LSMF (solid/dashed lines correspond to clockwise/counter-clockwise field direction).

polar regions (actually the field lines of the poloidal field are isocontours of the potential $A$, so the strength of the poloidal field is also seen). The dynamo oscillation closely resembles a standing wave.

\section{Conclusions}

We have studied kinematic axisymmetric mean-field dynamo models in which the $\boldsymbol{\Omega} \times \boldsymbol{J}$ effect was included as one of the mechanisms by which the large-scale magnetic field is generated. The effect was used in an extended form, together with all other effects that result from the combined action of rotation and a nonuniformity of the large-scale magnetic field, including anisotropic turbulent diffusion. Concurrently to the $\alpha$ effect, the $\boldsymbol{\Omega} \times \boldsymbol{J}$ effect may, in particular, generate the large-scale poloidal magnetic field of stars. Our results show that the inclusion of the additional effects alleviates some of the problems connected with the $\alpha$ effect and $\alpha \Omega$ dynamos. Compared to the standard $\alpha \Omega$ dynamo, the inclusion of the $\boldsymbol{\Omega} \times \boldsymbol{J}$ effect increases the time period of the dynamo and thus brings the models into better agreement with the solar observations. Furthermore, the large-scale toroidal field comes closer to the equator, bringing the models in better agreement with the observations also in this respect. The observed phase relation between the toroidal and poloidal field components is correctly reproduced and, in contrast to the $\alpha \Omega$ model, dynamo modes with the correct (dipolar) parity become unstable first.

We did not explore the possible effects of meridional circulation in the considered models. Circulation-dominated dynamo models are very popular presently. They apparently avoid the problems with $\alpha \Omega$ dynamos mentioned above. But they also introduce (yet) unproven assumptions concerning the meridional flow and work only if the turbulent magnetic diffusivity is reduced by about two orders of magnitude compared to the mixing-length estimates. We do not claim that the models considered in the present paper are superior to circulationdominated dynamo models. Possibly the effects studied here and meridional circulation should be combined. We have preliminary results (not published yet) which indicate that a $\delta \Omega$ dynamo model with meridional circulation, but without the $\alpha$ effect, admits oscillatory eigenmodes that give a solar-type dynamo. Another possible improvement of our model would be the inclusion of the tachocline.

We also gave a first example of a $\delta^{2}$ dynamo which is based solely on the joint induction effects of rotation and an inhomogeneity of the large-scale magnetic field, without differential rotation, the $\alpha$ effect and turbulent pumping. This kind of dynamo may be relevant for fully convective stars. We found a dynamo instability both for a strongly simplified model in plane geometry and for an axisymmetric model for a full sphere. The dynamo modes are steady if the effect of anisotropic diffusion is not included. In the case of the sphere, the inclusion of anisotropic diffusion yields a slowly oscillating magnetic field. Including turbulent pumping (due to density stratification, not part of a pure $\delta^{2}$ dynamo) leads to the same result. The period of the oscillation is on the order of the turbulent diffusion time of the system. For M dwarfs this is estimated to be 10-100 yr. Preliminary results for combinations of $\alpha^{2}$ and $\delta^{2}$ dynamo models (not contained in the paper and not yet published elsewhere) indicate that the mixture of the two effects generally produces oscillatory dynamo modes. Our aim here was not to provide a dynamo model for fully convective stars, but merely to suggest the combined effects of rotation and an inhomogeneity of the large-scale magnetic field as ingredients in future dynamo models.

Acknowledgements. The work of V. V. Pipin was supported by the Russian Foundation for Basis Research (RFBR) through grants 2258.2008.2 and 07-0200246.

\section{Appendix}

Here we give the definitions of the functions $f_{i}^{(a)}$ and $f_{i}^{(d)}$ that are used in the representation of the turbulent electromotive force $\mathcal{E}$. For details of the calculations we refer to Pipin (2008).

$$
\begin{aligned}
f_{1}^{(a)}= & \frac{1}{4 \Omega^{* 2}}\left[\left(\Omega^{* 2}+3\right) \frac{\arctan \Omega^{*}}{\Omega^{*}}-3\right] \\
f_{2}^{(a)}= & \frac{1}{4 \Omega^{* 2}}\left[\left(\Omega^{* 2}+1\right) \frac{\arctan \Omega^{*}}{\Omega^{*}}-1\right] \\
f_{3}^{(a)}= & \frac{1}{4 \Omega^{* 2}}\left[\left((\varepsilon-1) \Omega^{* 2}+\varepsilon-3\right) \frac{\arctan \Omega^{*}}{\Omega^{*}}+3-\varepsilon\right] \\
f_{4}^{(a)}= & \frac{1}{6 \Omega^{* 3}}\left[3\left(\Omega^{* 4}+6 \varepsilon \Omega^{* 2}+10 \varepsilon-5\right) \frac{\arctan \Omega^{*}}{\Omega^{*}}\right. \\
& \left.-\left((8 \varepsilon+5) \Omega^{* 2}+30 \varepsilon-15\right)\right], \\
f_{5}^{(a)}= & \frac{1}{3 \Omega^{* 3}}\left[3\left(\Omega^{* 4}+3 \varepsilon \Omega^{* 2}+5(\varepsilon-1)\right) \frac{\arctan \Omega^{*}}{\Omega^{*}}\right. \\
& \left.-\left((4 \varepsilon+5) \Omega^{* 2}+15(\varepsilon-1)\right)\right] \\
f_{6}^{(a)}= & -\frac{1}{48 \Omega^{* 3}}\left[3\left((3 \varepsilon-11) \Omega^{* 2}+5 \varepsilon-21\right) \frac{\arctan \Omega^{*}}{\Omega^{*}}\right. \\
& \left.-\left(4(\varepsilon-3) \Omega^{* 2}+15 \varepsilon-63\right)\right],
\end{aligned}
$$




$$
\begin{aligned}
f_{7}^{(a)}= & \frac{1}{48 \Omega^{* 3}}\left[3\left((5 \varepsilon+3) \Omega^{* 2}+11 \varepsilon+5\right) \frac{\arctan \Omega^{*}}{\Omega^{*}}\right. \\
& \left.-\left(4(\varepsilon+1) \Omega^{* 2}+33 \varepsilon+15\right)\right], \\
f_{8}^{(a)}= & -\frac{1}{12 \Omega^{* 3}}\left[3\left((3 \varepsilon+1) \Omega^{* 2}+4 \varepsilon-2\right) \frac{\arctan \Omega^{*}}{\Omega^{*}}\right. \\
& \left.-\left(5(\varepsilon+1) \Omega^{* 2}+12 \varepsilon-6\right)\right], \\
f_{9}^{(a)}= & \frac{\varepsilon+1}{4 \Omega^{*}}\left(\frac{\arctan \Omega^{*}}{\Omega^{*}}-1\right), \\
f_{10}^{(a)}= & \frac{1}{3 \Omega^{* 3}}\left[3\left(\Omega^{* 2}+1\right)\left(\Omega^{* 2}+\varepsilon-1\right) \frac{\arctan \Omega^{*}}{\Omega^{*}}\right. \\
& \left.-\left((2 \varepsilon+1) \Omega^{* 2}+3 \varepsilon-3\right)\right], \\
f_{4}^{(d)}= & \frac{1}{2 \Omega^{* 3}}\left[\left(2 \Omega^{* 2}+3\right)-3\left(\Omega^{* 2}+1\right) \frac{\arctan \left(\Omega^{*}\right)}{\Omega^{*}}\right] . \\
f_{3}^{(d)}= & \frac{1}{2 \Omega^{* 3}}\left[3\left(3 \Omega^{* 2}+5\right) \frac{\arctan \left(\Omega^{*}\right)}{\Omega^{*}}-\left(4 \Omega^{* 2}+15\right),\right. \\
f_{11}^{(a)}= & \frac{1}{6 \Omega^{* 3}}\left[3\left(\Omega^{* 2}+1\right)\left(\Omega^{* 2}+2 \varepsilon-1\right) \frac{\arctan \Omega^{*}}{\Omega^{*}}\right. \\
f_{2}^{(d)}= & \frac{1}{4 \Omega^{* 2}}\left[\left((\varepsilon-1) \Omega^{* 2}+3 \varepsilon+1\right) \frac{\arctan \left(\Omega^{*}\right)}{\Omega^{*}}\right. \\
& \left.-\left((4 \varepsilon+1) \Omega^{* 2}+6 \varepsilon-3\right)\right] . \\
f_{1}^{(d)}= & \frac{1}{2 \Omega^{* 3}}\left[(\varepsilon+1) \Omega^{* 2}+3 \varepsilon\right. \\
& \left.-\left((2 \varepsilon+1) \Omega^{* 2}+3 \varepsilon\right) \frac{\left.\arctan \left(\Omega^{*}\right)\right]}{\Omega^{*}}\right],
\end{aligned}
$$

\section{References}

Boyd, J. P. 2001, Chebyshev and Fourier Spectral Methods, 2nd Ed. (Mineola, NY: Dover Publications)

Brandenburg, A. 2005, ApJ, 625, 539
Brandenburg, A., \& Subramanian, K. 2005, Phys. Rep., 417, 1

Browning, M. K. 2008, ApJ, 676, 1262

Chabrier, G., \& Küker, M. 2006, A\&A, 446, 1027

Dikpati, M., \& Gilman, P. A. 2007, New J. Phys., 9, 297

Dikpati, M., Rempel, M., Gilman, P. A., \& MacGregor, K. B. 2005, A\&A, 437, 699

Dobler, W., Stix, M., \& Brandenburg, A. 2006, ApJ, 638, 336

Eggleton, P. P. 1971, MNRAS, 151, 351

Eggleton, P. P., \& Kiseleva-Eggleton, L. 2002, ApJ, 575, 461

Elstner, D., \& Rüdiger, G. 2007, Astron. Nachr., 328, 1130

Galloway, D. J., \& Weiss, N. O. 1981, ApJ, 243, 945

Godier, S., \& Rozelot, J.-P. 2000, A\&A, 355, 365

Guerrero, G., \& de Gouveia Dal Pino, E. M. 2008, A\&A, 485, 267

Izzard, R. G., \& Glebbeek, E. 2006, New A, 12, 161

Jouve, L., Brun, A. S., Arlt, R., et al. 2008, A\&A, 483, 949

Kitchatinov, L. L. 2002, A\&A, 394, 1135

Kitchatinov, L. L. 2004, in NATO Science Series II: Mathematics, Physics and Chemistry, 124, Turbulence, Waves and Instabilities in the Solar Plasma, ed. R. Erdélyi, K. Petrovay, B. Roberts, \& M. J. Aschwanden (Dordrecht: Kluwer), 81

Kichatinov, L. L., \& Pipin, V. V. 1993, A\&A, 274, 647

Kitchatinov, L. L., Pipin, V. V., \& Rüdiger, G. 1994, Astron. Nachr., 315, 157

Köhler, H. 1973, A\&A, 25, 467

Krause, F., \& Rädler, K.-H. 1980, Mean-Field Magnetohydrodynamics and Dynamo Theory (Berlin: Akademie-Verlag)

Küker, M., \& Rüdiger, G. 1999, A\&A, 346, 922

Kuzanyan, K. M., Pipin, V. V., \& Seehafer, N. 2006, Sol. Phys., 233, 185

Ossendrijver, M. 2003, A\&A Rev., 11, 287

Parker, E. N. 1955, ApJ, 122, 293

Parker, E. N. 1979, Cosmical Magnetic Fields (Oxford: Clarendon Press)

Pipin, V. V. 2007, Astron. Rep., 51, 411

Pipin, V. V. 2008, Geophys. Astrophys. Fluid Dynam., 102, 21

Rädler, K.-H. 1969, Monatsber. Dtsch. Akad. Wiss. Berlin, 11, 194, in German, English translation in ed. P. H. Roberts, \& M. Stix, Report No. NCAR-TN/IA$60(1971)$

Rädler, K.-H. 1980, Astron. Nachr., 301, 101

Rädler, K.-H. 2000, in From the Sun to the Great Attractor: 1999 Guanajuato Lectures on Astrophysics, ed. D. Page, \& J. G. Hirsch (New York: Springer), Lecture Notes in Physics, 556, 101

Rädler, K.-H., \& Rheinhardt, M. 2007, Geophys. Astrophys. Fluid Dynam., 101, 117

Rädler, K.-H., \& Stepanov, R. 2006, Phys. Rev. E, 73, 056311

Rädler, K.-H., Kleeorin, N., \& Rogachevskii, I. 2003, Geophys. Astrophys. Fluid Dynam., 97, 249

Rogachevskii, I., \& Kleeorin, N. 2003, Phys. Rev. E, 68, 036301

Rogachevskii, I., \& Kleeorin, N. 2004, Phys. Rev. E, 70, 046310

Rüdiger, G., \& Hollerbach, R. 2004, The Magnetic Universe (Weinheim: Wiley-VCH)

Schou, J., Antia, H. M., Basu, S., et al. 1998, ApJ, 505, 390

Schüssler, M. 1980, Nature, 288, 150

Schüssler, M., \& Ferriz-Mas, A. 2003, in Advances in Nonlinear Dynamos, ed. A. Ferriz-Mas, \& M. Núñez (London: Taylor \& Francis), 123

Seehafer, N., Gellert, M., Kuzanyan, K. M., \& Pipin, V. V. 2003, Adv. Space Res., 32, 1819

Spiegel, E. A., \& Weiss, N. O. 1980, Nature, 287, 616

Steenbeck, M., Krause, F., \& Rädler, K.-H. 1966, Z. Naturforsch., 21a, 369

Stix, M. 1976, A\&A, 47, 243

Stix, M. 2002, The Sun. An Introduction, 2nd Ed. (Berlin: Springer)

Yoshimura, H. 1975, ApJ, 201, 740 